\title{
THE CONGRUENCE LATTICE OF AN IDEAL EXTENSION OF SEMIGROUPS
}

by MARIO PETRICH

\author{
Dedicated to the memory of Ottó Steinfeld \\ (Received 20 June, 1991; revised 26 March, 1992)
}

1. Introduction and summary. Let $S$ be an ideal of a semigroup $V$. In such a case, $V$ is an (ideal) extension of $S$ by $T=V / S$. The problem considered in [2] is the construction of all congruences on $V$ in terms of congruences on $S$ and $T$. This did not succeed for all congruences but it did for those congruences whose restriction to $S$ is weakly reductive. If the extension is strict, more precise constructions are also given there. With some relatively weak restrictions on $S$, we are able to obtain in this way all congruences on $V$ in the form indicated above.

When both $S$ and $T$ are completely 0 -simple, a study of the congruence lattice $\mathscr{C}(V)$ of $V$ in terms of those of $S$ and $T$ can be found in [4]. In that paper, meets and joins of congruences on $V$ in this form are described, the relations on $\mathscr{C}(V)$ induced by restrictions of congruences on $V$ to $S$ and to $T^{*}$.

In this paper we consider the middle ground: $V$ is an extension of $S$ by $T$ and all congruences on $S$ are weakly reductive, or both $S$ and $T$ are regular, and under these circumstances we study the congruence lattice $\mathscr{C}(V)$. This is a slight narrowing of the hypothesis in the first paragraph above but a large widening of the hypotheses in the second paragraph. We thus cannot hope to generalize all the results in [4] to our present situation.

Section 2 contains all the preliminaries and serves to fix the notation used. The inclusion relation, meets and joins are provided in Section 3 . The relation on the congruence lattice induced by restriction of congruences to the ideal is studied in Section 4. The special situation arising when the semigroup is regular and the ideal is completely 0 -simple is considered in the final Section 5.

Our study is not complete. We have navigated carefully among the rocks and boulders representing the substantial difficulties arising from the relative incompatibility of the concepts under study. We have succeeded in providing a frame for a possibly more profound study of the congruence lattice $\mathscr{C}(V)$ in terms of the congruence lattices $\mathscr{C}(S)$ and $\mathscr{C}(T)$. The main difficulty in such a study is the extra element, which is apparently restricted to neither $\mathscr{C}(S)$ nor $\mathscr{C}(T)$, of the saturation of the ideal $S$ by a congruence on $V$. When two congruences on $V$ are given, it is the interplay of their saturations of $S$ that causes the most serious complications. We nevertheless believe that this study has a future offering sufficient reward.

2. Preliminaries. We employ the standard notation and terminology which can be found, for example, in [3]. For emphasis, or in addition, we state explicitly the following nomenclature and symbolism.

The equality and the universal relations on a set $X$ are denoted by $\varepsilon_{X}$ and $\omega_{X}$, respectively. The restriction of a function or a relation $\theta$ to a set $X$ is denoted by $\theta_{\mid X}$. If $\theta$ is an equivalence relation on a set $X$ and $x \in X$, then $x \theta$ denotes the $\theta$-class containing $x$; 
if $A \subseteq X$ and

$$
A=\{x \in X \mid x \theta a \text { for some } a \in A\},
$$

then $\theta$ saturates $A$. If $A$ and $B$ are any sets, then

$$
A \backslash B=\{a \in A \mid a \notin B\} .
$$

If $\alpha$ and $\beta$ are elements of a lattice $L$ such that $\alpha \leq \beta$, then

$$
[\alpha, \beta]=\{\gamma \in L \mid \alpha \leq \gamma \leq \beta\} .
$$

Let $Q$ be a semigroup. If $A \subseteq Q$, then its set of idempotents is denoted by $E(A)$. If $Q$ has an identity, then $Q^{1}=Q$ otherwise $Q^{1}$ stands for $Q$ with an identity adjoined. The congruence lattice of $Q$ is denoted by $\mathscr{C}(Q)$. If $Q$ has a zero and $A \subseteq Q$, then $A^{*}=A \backslash\{0\}$; an equivalence relation $\theta$ on $Q$ having $\{0\}$ as a class is 0 -restricted; the set of all 0-restricted congruences on $Q$ is denoted by $\mathscr{C}_{0}(Q)$. If $\theta$ is a relation on $Q, \theta^{*}$ denotes the congruence on $Q$ generated by $\theta$. If $\theta$ is an equivalence relation on $Q$, then $\theta^{\circ}$ denotes the greatest congruence on $Q$ contained in $\theta$; explicitly

$$
a \theta^{\circ} b \Leftrightarrow x a y \theta x b y \text { for all } x, y \in Q^{1} .
$$

Of particular importance is the special case: for a subset $A$ of $Q$, and $\theta$ the equivalence relation with classes $A$ and $Q \backslash A$ (whichever is nonempty), $\pi_{A}=\theta^{\circ}$ is the principal congruence on $Q$ relative to $A$; explicitly

$$
a \pi_{A} b \Leftrightarrow\left(x a y \in A \Leftrightarrow x b y \in A \text { for all } x, y \in Q^{1}\right) .
$$

In fact, $\pi_{A}$ is the greatest congruence on $Q$ which saturates $A$. In the special case when $Q$ has a zero, $\xi_{Q}=\pi_{\{0\}}$ is the greatest 0-restricted congruence on $Q$. The semigroup $Q$ is weakly reductive if for any $a, b \in Q, a x=b x$ and $x a=x b$ for all $x \in Q$ implies $a=b$. We say that a congruence $\rho$ on $Q$ is weakly reductive if $Q / \rho$ is weakly reductive. If $R$ is an ideal of $Q$, then $Q / R$ denotes the Rees quotient semigroup of $Q$ relative to $R$; as a set, $Q / R=(Q \backslash R) \cup\{0\}$.

Throughout the paper we fix the following notation: $V$ is an (ideal) extension of $S$ by $T$, that is $S$ is an ideal of $V$ and the Rees quotient $V / S \cong T$, where we take that $V=S \cup T^{*}$. In order to simplify our statements, we assume that all congruences on $S$ are weakly reductive. For example this is true if each element of $S$ has a left and a right identity for in that case this carries over to all homomorphic images and implies weak reductivity ([1, Lemma 1]). Weak reductivity of $Q$ is not sufficient for each congruence on $Q$ to be weakly reductive as the example in [2] shows. A sufficient condition for this is regularity. If there exists a partial homomorphism $\varphi: T^{*} \rightarrow S$ such that for any $a, b \in T^{*}$ and $x \in S$,

$$
a x=(a \varphi) x, \quad x a=x(a \varphi), \quad a b=(a \varphi)(b \varphi) \quad \text { if } \quad a b \in S,
$$

then we say that $\varphi$ determines the multiplication of $V$ and $V$ is a strict extension of $S$.

3. Meets and joins. From [2, Corollary 1 to Theorem 1] we deduce the following description of congruences on $V$. Let $\sigma \in \mathscr{C}(S), P$ be an ideal of $T, \tau \in \mathscr{C}_{0}(T / P)$ satisfying 
the following conditions:

(i) $a, b \in T \backslash P, a \tau b, x \sigma y \Rightarrow a x \sigma b y, x a \sigma y b$,

(ii) for every $a \in P^{*}$ there exists $a^{\prime} \in S$ such that $x \in S \Rightarrow a x \sigma a^{\prime} x, x a \sigma x a^{\prime}$.

In such a case we say that $a$ and $a^{\prime}$ are $\sigma$-linked, call $(\sigma, P, \tau)$ an admissible triple and define a relation $v$ on $V$ by

$$
a v b \Leftrightarrow \begin{cases}a \tau b, & \text { if } a, b \in T \backslash P, \\ a^{\prime} \sigma b^{\prime} & \text { if } a, b \in P^{*}, \\ a^{\prime} \sigma b & \text { if } a \in P^{*}, b \in S, \\ a \sigma b^{\prime} & \text { if } a \in S, b \in P^{*}, \\ a \sigma b & \text { if } a, b \in S\end{cases}
$$

where $a, a^{\prime}$ and $b, b^{\prime}$ are $\sigma$-linked. Then $v$ is a congruence on $V$ and, conversely, every congruence on $V$ has this form. We shall see in Corollary 3.2 that this representation is unique. The notation $v=\mathscr{C}(\sigma, P, \tau)$ will always denote such a congruence on $V$ implicitly implying that $(\sigma, P, \tau)$ is an admissible triple. In fact, given $v \in \mathscr{C}(V)$, the admissible triple for $v$ is $(\sigma, P, \tau)$, where

$$
\sigma=v_{\mid S}, \quad P=\left\{a \in T^{*} \mid a v b \text { for some } b \in S\right\}, \quad a \tau b \Leftrightarrow a, b \in T \backslash P, a v b \text { and } 0 \tau 0 \text {. }
$$

We first establish necessary and sufficient conditions for the inclusion of two congruences in the above representation. A technical lemma is needed for the representation of the meet of two such congruences. We conclude the section with the analogous representation of the join.

Lemma 3.1. Let $v_{i}=\mathscr{C}\left(\sigma_{i}, P_{i}, \tau_{i}\right)$ for $i=1,2$. Then $v_{1} \subseteq v_{2}$ if and only if

(i) $\sigma_{1} \subseteq \sigma_{2}$,

(ii) $P_{1} \subseteq P_{2}$,

(iii) $\tau_{1}$ saturates $P_{2} \backslash P_{1}$,

(iv) $\tau_{1 \mid \pi P_{2}} \subseteq \tau_{2 \mid \lambda P_{2}}$.

Proof. Necessity. (i) If $a \sigma_{1} b$, the $a v_{1} b$ so by hypothesis $a v_{2} b$ and thus $a \sigma_{2} b$. Therefore $\sigma_{1} \subseteq \sigma_{2}$.

(ii) If $a \in P_{1}^{*}$, then $a v_{1} b$ for some $b \in S$ so by hypothesis $a v_{2} b$ and thus $a \in P_{2}^{*}$. Therefore $P_{1} \subseteq P_{2}$.

(iii) If $a \in P_{2}^{*}, b \in T \backslash P_{1}$ and $a \tau_{1} b$, then $a v_{1} b$ so by hypothesis $a v_{2} b$ whence $b \in P_{2}^{*}$, since $v_{2}$ saturates $S \cup P_{2}^{*}$. Therefore $\tau_{1}$ saturates $P_{2} \backslash P_{1}$.

(iv) If $a, b \in T \backslash P_{2}$ are such that $a \tau_{1} b$, then $a v_{1} b$ so by hypothesis $a v_{2} b$ and thus $a \tau_{2} b$. Therefore $\tau_{1 \mid \lambda v_{2}} \subseteq \tau_{2 \mid \wedge p_{2}}$.

Sufficiency. Let $a v_{1} b$. If $a, b \in S$, then $a \sigma_{1} b$ and (i) gives $a \sigma_{2} b$ so that $a v_{2} b$. Let $a \in P_{1}^{*}$ and $b \in S$. Then there exists $a^{\prime} \in S$ which is $\sigma_{1}$-linked to $a$ and thus also $\sigma_{2}$-linked to $a$ by (i). Also $a^{\prime} \sigma_{1} b$ which then gives $a^{\prime} \sigma_{2} b$ again by (i). It follows that $a v_{2} b$. The case $a \in S$ and $b \in P_{1}^{*}$ is dual. Let $a, b \in P_{1}^{*}$. Then with the same notation for $a^{\prime}$ and the analogous one for $b^{\prime}$, we obtain $a^{\prime} \sigma_{2} b^{\prime}$ so that $a v_{2} b$. Next let $a, b \in P_{2} \backslash P_{1}$. Then $a \tau_{1} b$ and $a, a^{\prime}$ and $b, b^{\prime}$ are $\sigma_{2}$-linked for some $a^{\prime}, b^{\prime} \in S$. For any $x \in S$, we get $a x \sigma_{1} b x$ and $x a \sigma_{1} x b$. By (i), we get $a x \sigma_{2} b x$ and $x a \sigma_{2} x b$. Since also $a x \sigma_{2} a^{\prime} x, x a \sigma_{2} x a^{\prime}, b x \sigma_{2} b^{\prime} x$, $x b \sigma_{2} x b^{\prime}$, we obtain $a^{\prime} x \sigma_{2} b^{\prime} x$ and $x a^{\prime} \sigma_{2} x b^{\prime}$. This holds for all $x \in S$, so by weak 
reductivity of $S / \sigma_{2}$, we conclude that $a^{\prime} \sigma_{2} b^{\prime}$ and thus $a v_{2} b$. If $a, b \in T \backslash P_{2}$, then $a \tau_{1} b$ which by (iv) implies that $a \tau_{2} b$ so that $a v_{2} b$. Since $v_{1}$ saturates $S \cup P_{1}^{*}$, and in view of (iii), it also saturates $S \cup P_{2}^{*}$, we have exhausted all the cases. Therefore $v_{1} \subseteq v_{2}$.

Corollary 3.2. Let $v_{i}=\mathscr{C}\left(\sigma_{i}, P_{i}, \tau_{i}\right)$ for $i=1,2$. Then $v_{1}=v_{2}$ if and only if $\sigma_{1}=\sigma_{2}$, $P_{1}=P_{2}, \tau_{1}=\tau_{2}$.

This corollary shows the uniqueness of the representation $\mathscr{C}(\sigma, P, \tau)$. Our next task is to start with two congruences on $V$ so represented and find the representation of their meet and join. It is somewhat surprising that the meet is harder to construct than the join. For the former, we first establish a lemma which takes care of the greater part of the proof. Recall that $\zeta_{Q}$ denotes the greatest 0 -restricted congruence on a semigroup $Q$ with zero. It will be helpful to keep in mind the following simple result.

Lemma 3.3. Let $Q$ be an ideal of $T$.

(i) $\left(\omega_{S}, Q, \xi_{T / Q}\right)$ is an admissible triple.

(ii) If $(-, Q,-)$ is any admissible triple, then $\mathscr{C}(-, Q,-) \subseteq \mathscr{C}\left(\omega_{S}, Q, \xi_{T / Q}\right)$.

Proof. (i) This is trivial.

(ii) This follows from Lemma 3.1 since $\omega_{S}$ is the greatest congruence on $S$ and $\xi_{T / Q}$ is the greatest 0 -restricted congruence on $T / Q$.

Lemma 3.4. Let $v=\mathscr{C}(\sigma, P, \tau)$, and let $Q$ be an ideal of $T$ contained in $P$.

(i) Define a relation $\sigma^{\prime}$ on $P / Q$ by

$$
a \sigma^{\prime} b \Leftrightarrow a x \sigma b y, x a \sigma y b \text { for all } x \sigma y
$$

if $a, b \in P \backslash Q$, and define $0 \sigma^{\prime} 0$. Then $\sigma^{\prime}$ is a 0 -restricted equivalence relation on $P / Q$. Let $\bar{\sigma}=\left(\sigma^{\prime}\right)^{\circ}$ in $P / Q ;$ then $\bar{\sigma} \in \mathscr{C}_{0}(P / Q)$.

(ii) Define a relation $(\sigma, \tau)$ on $T / Q$ by

$$
a(\sigma, \tau) b \Leftrightarrow \begin{cases}a \tau b & \text { if } a, b \in T \backslash P, \\ a \bar{\sigma} b & \text { if } a, b \in P \backslash Q, \\ a=b=0 & \text { otherwise. }\end{cases}
$$

Then $(\sigma, \tau) \in \mathscr{C}_{0}(T / Q)$ and both $(\sigma, Q,(\sigma, \tau))$ and $\left(\omega_{S}, Q, \xi_{T / Q}\right)$ are admissible triples. Let $\hat{v}=\mathscr{C}(\sigma, Q,(\sigma, \tau))$.

(iii) $\hat{v}=v \wedge \mathscr{C}\left(\omega_{S}, Q, \xi_{T / Q}\right)$.

Proof. (i) Let $a \in P \backslash Q$. By hypothesis, there exists $a^{\prime} \in S$ which is $\sigma$-linked to $a$. Hence for any $x \sigma y$, we obtain $a x \sigma a^{\prime} x \sigma a^{\prime} y \sigma a y$ so that $a x \sigma a y$ and similarly $x a \sigma y a$. Thus $\sigma^{\prime}$ is reflexive and it is obviously symmetric. Let $a, b, c \in P \backslash Q$ be such that $a \sigma^{\prime} b$ and $b \sigma^{\prime} c$. For $x \sigma y$, we get $a x \sigma b y \sigma c y, x a \sigma y b \sigma y c$ and thus $a \sigma^{\prime} c$. Therefore $\sigma^{\prime}$ is also transitive and is thus an equivalence relation on $P / Q$. Hence $\bar{\sigma}=\left(\sigma^{\prime}\right)^{\circ}$ is defined and since $\sigma^{\prime}$ is 0 -restricted, so is $\bar{\sigma}$.

(ii) Since $P$ is an ideal of $T, P / Q$ is an ideal of $T / Q$. Now $\bar{\sigma}$ is a congruence on $P / Q$ and $\tau$ is a 0 -restricted congruence on $T / P$ and hence also on $(T / Q) /(P / Q)$. In order to prove that $(\sigma, \tau)$ is a congruence on $T / Q$, we apply the definition. Hence let $a, b \in T \backslash P$ and $x, y \in P \backslash Q$ be such that $a \tau b$ and $x \bar{\sigma} y$; we must show that $a x \bar{\sigma} b y$ and $x a \bar{\sigma} y b$. Let $u, v \in(P / Q)^{1}$. Then $u x v \bar{\sigma} u y v$ and hence $u x v \neq 0$ if and only if $u y v \neq 0$ in $P / Q$. Assume 
that $u x v \neq 0$ in $P / Q$. For any $s \sigma t$, we get $(u x v) s \sigma(u y v) t$ and $s(u x v) \sigma t(u y v)$. If $u \in P \backslash Q$, then for some $u^{\prime} \in S$, we have that $u$ and $u^{\prime}$ are $\sigma$-linked. If $u=1$, let $u^{\prime}=1$. With this convention and the above notation, we obtain

and

$$
\begin{array}{rlrl}
{[u(a x) v] s} & =[(u a) x v] s \sigma[(u a) y v] t=u a(y v t) \sigma u^{\prime} a(y v t) \\
& \sigma u^{\prime} b(y v t) & \left.\left(\text { since } u^{\prime}=1 \text { or } u^{\prime} \in S\right), y v t \in S, a \tau b\right) \\
& \sigma u b(y v t) & (\text { since } b(y v t) \in S) \\
& =[u(b y) v] t &
\end{array}
$$

$$
\begin{aligned}
& s[u(a x) v]=s[(u a) x v] \sigma t[(u a) y v] \\
& = \begin{cases}(t u a) y v \sigma(t u a) y^{\prime} & \text { if } v=1 \\
(t u a) t v \sigma(t u a) y v^{\prime} & \text { otherwise }\end{cases} \\
& = \begin{cases}(t u) a y^{\prime} \sigma(t u) b y^{\prime} & \text { if } \left.v=1 \text { (since } t u, y^{\prime} \in S\right) \\
(t u) a\left(y v^{\prime}\right) \sigma(t u) b\left(y v^{\prime}\right) & \text { otherwise (since } \left.t u, y v^{\prime} \in S\right)\end{cases} \\
& \sigma t[u(b y) v] .
\end{aligned}
$$

This proves that $u(a x) v \sigma^{\prime} u(b y) v$ and thus $a x \bar{\sigma} b y$. One shows similarly that $x a \bar{\sigma} y b$.

Therefore $(\sigma, \tau) \in \mathscr{C}(T / Q)$. Since $\bar{\sigma}$ is 0 -restricted, so is $(\sigma, \tau)$ and thus $(\sigma, \tau) \epsilon$ $\mathscr{C}_{0}(T / Q)$. Further, $Q \subseteq P$ shows that every element in $Q^{*}$ is $\sigma$-linked to some element of $S$. If $a, b \in T \backslash Q$ are such that $a(\sigma, \tau) b$ and $x \sigma y$, then either $a \tau b$ or $a \bar{\sigma} b$ and the desired conclusion $a x \sigma b y, x a \sigma y b$ follows in the first case by hypothesis and in the second case by the definition of $\bar{\sigma}$. Therefore $(\sigma, Q,(\sigma, \tau))$ is an admissible triple and we may let $\hat{v}=\mathscr{C}(\sigma, Q,(\sigma, \tau))$. A similar argument will show that also $\left(\omega_{S}, Q, \xi_{T / Q}\right)$ is an admissible triple.

(iii) Let $a \hat{v} b$. If $a, b \in S \cup Q^{*}$ or $a, b \in T \backslash P$, then clearly $a v b$. It remains to consider the case $a, b \in P \backslash Q$. In this case $a \bar{\sigma} b$ whence $a \sigma^{\prime} b$. Hence for any $x \sigma y$, we have $a x \sigma b y$ and $x a \sigma y b$. Since $a, b \in P^{*}$, there exist $a^{\prime}, b^{\prime} \in S$ such that $a, a^{\prime}$ and $b, b^{\prime}$ are $\sigma$-linked. It follows that for all $x \in S$,

$$
a x \sigma a^{\prime} x, x a \sigma x a^{\prime}, b x \sigma b^{\prime} x, x b \sigma x b^{\prime}
$$

which then implies that $a^{\prime} x \sigma b^{\prime} x$ and $x a^{\prime} \sigma x b^{\prime}$. Since $S / \sigma$ is weakly reductive, we conclude that $a^{\prime} \sigma b^{\prime}$ which proves that $a v b$. Therefore $\hat{v} \subseteq v$. Since $\xi_{T / Q}$ is the greatest 0 -restricted congruence on $T / Q$, we get $(\sigma, \tau) \subseteq \xi_{T / Q}$. It then follows that

$$
\hat{v}=\mathscr{C}(\sigma, Q,(\sigma, \tau)) \subseteq \mathscr{C}\left(\omega_{S}, Q, \xi_{\tau / Q}\right) .
$$

For the opposite inclusion, let $(a, b) \in v \wedge \mathscr{C}\left(\omega_{S}, Q, \xi_{T / Q}\right)$. Then either $a, b \in S \cup Q^{*}$ or $a, b \in P \backslash Q$ or $a, b \in T \backslash P$. In the first and last case, clearly $a \hat{v} b$. We consider the case $a, b \in P \backslash Q$. Let $u, v \in(P / Q)^{1} \backslash\{0\}$. Then $u a v v u b v$ so either $u a v, u b v \in P \backslash Q$ or $u a v=$ $u b v=0$ in $P / Q$. Assume the former. For any $x \sigma y$, we have $(u a v) x \sigma(u b v) y$ and $x(u a v) \sigma y(u b v)$. It follows that uav $\sigma^{\prime} u b v$ whence $a \bar{\sigma} b$ and finally $a \hat{v} b$. Therefore $a \hat{v} b$ in all cases which proves that $v \wedge \mathscr{C}\left(\omega_{S}, Q, \xi_{T / Q}\right) \subseteq \hat{v}$ and equality prevails.

We can now easily derive the expression for the meet. 
TheOREM 3.5. Let $v_{i}=\mathscr{C}\left(\sigma_{i}, P_{i}, \tau_{i}\right)$ for $i=1,2$. Then

$$
v_{1} \wedge v_{2}=\hat{v}_{1} \wedge \hat{v}_{2}=\mathscr{C}(\sigma, P, \tau)
$$

where

$$
\begin{aligned}
& \sigma=\sigma_{1} \wedge \sigma_{2} \\
& P=\left\{a \in P_{1}^{*} \cap P_{2}^{*} \mid a \text { is } \sigma \text {-linked to an element of } S\right\} \cup\{0\} \\
& \hat{v}_{i}=\mathscr{C}\left(\sigma_{i}, P,\left(\sigma_{i}, \tau_{i}\right)\right) \in \mathscr{C}(V) \text { for } i=1,2, \\
& \tau=\left(\sigma_{1}, \tau_{1}\right) \wedge\left(\sigma_{2}, \tau_{2}\right) .
\end{aligned}
$$

Proof. By Lemma 3.4(iii), we obtain

$$
\begin{aligned}
\hat{v}_{1} \wedge \hat{v}_{2} & =\left[v_{1} \wedge \mathscr{C}\left(\omega_{S}, P, \xi_{T / P}\right)\right] \wedge\left[v_{2} \wedge \mathscr{C}\left(\omega_{S}, P, \xi_{T / P}\right)\right] \\
& =\left(v_{1} \wedge v_{2}\right) \wedge \mathscr{C}\left(\omega_{S}, P, \xi_{T / P}\right) \\
& =v_{1} \wedge v_{2} \quad\left(\text { since } v_{1} \wedge v_{2} \subseteq \mathscr{C}\left(\omega_{S}, P, \xi_{T / P}\right)\right) .
\end{aligned}
$$

This proves the first equality; the second follows easily by considering the cases: $a, b \in S$; $a \in S, b \in P^{*} ; a \in P^{*}, b \in S ; a, b \in P^{*}$ and $a, b \in T \backslash P$.

We can now pass directly to the representation of the join.

THEOREM 3.6. Let $v_{i}=\mathscr{C}\left(\sigma_{i}, P_{i}, \tau_{i}\right)$ for $i=1,2$. Then $v_{1} \vee v_{2}=\mathscr{C}(\sigma, P, \tau)$ where $\sigma=\sigma_{1} \vee \sigma_{2}, P=\left(P_{1} \cup P_{2}\right)\left(\tau_{1} \vee \tau_{2}\right)$ and $\tau$ is the 0-restricted congruence on $T / P$ satisfying the condition $\tau_{\mid \pi P}=\left(\tau_{1} \vee \tau_{2}\right)_{\mid r P}$.

Proof. 1. $P$ is an ideal of $T$. Let $a \in P^{*}$ and $b \in T^{*}$ and assume that $a b \notin P$. Then there exists a sequence either of the form

$$
a \tau_{1} a_{1} \tau_{2} a_{2} \ldots a_{n-1} \tau_{2} a_{n} \in P_{1}^{*}
$$

or of the form

$$
a \tau_{1} a_{1} \tau_{2} a_{2} \ldots a_{n-1} \tau_{1} a_{n} \in P_{2}^{*},
$$

for we may set $a=a_{1}$ if necessary. By symmetry, we may consider only the first case. Since $a b \notin P_{1}$, we have $a b \tau_{1} a_{1} b \notin P_{1}$. If $a_{1} b \in P_{2}$, then $a b \in P_{1} \tau_{2} \subseteq P$, contradicting the hypothesis. Hence $a_{1} b \notin P_{2}$ so $a_{1} b \tau_{2} a_{2} b \notin P_{2}$. Now we similarly conclude that $a_{2} b \notin P_{1}$ and continuing this process, we arrive at the sequence

$$
a b \tau_{1} a_{1} b \tau_{2} a_{2} b \ldots a_{n-1} b \tau_{2} a_{n} b \in P_{1}^{*}
$$

which gives $a b \in P_{1} \tau_{2} \ldots \tau_{1} \subseteq P$, contradicting the hypothesis. We thus conclude that $a b \in P$. Similarly we get $b a \in P$ which proves that $P$ is an ideal of $T$.

2. Every element of $P^{*}$ is $\sigma_{1} \vee \sigma_{2}$-linked to some element of $S$. Let $a \in P^{*}$. Again we have the two possibilities (1) and (2) and we may consider the first. There is $a^{\prime} \in S$ such that $a_{n}$ and $a^{\prime}$ are $\sigma_{1}$-linked. For any $x \in S$, we have

$$
\begin{aligned}
& a x \sigma_{1} a_{1} x \sigma_{2} a_{2} x \ldots a_{n-1} x \sigma_{2} a_{n} x \sigma_{1} a^{\prime} x, \\
& x a \sigma_{1} x a_{1} \sigma_{2} x a_{2} \ldots x a_{n-1} x \sigma_{2} x a_{n} \sigma_{1} x a^{\prime}
\end{aligned}
$$

which implies that $a x \sigma_{1} \vee \sigma_{2} a^{\prime} x$ and $x a \sigma_{1} \vee \sigma_{2} x a^{\prime}$ so that $a$ and $a^{\prime}$ are $\sigma_{1} \vee \sigma_{2}$-linked.

3. $(\sigma, P, \tau)$ is an admissible triple. Clearly $\tau_{1} \vee \tau_{2}$ saturates $P$ and hence $\tau$ can be defined as the 0 -restricted congruence on $T / P$ satisfying the condition in the statement of 
the theorem. Let $a, b \in T \backslash P$ be such that $a \tau b$ and let $x \sigma y$. Then $a \tau_{1} \vee \tau_{2} b$ and $x \sigma_{1} \vee \sigma_{2} y$ so that there exist sequences

$$
a \tau_{1} c_{1} \tau_{2} c_{2} \ldots c_{m} \tau_{2} b, \quad x \sigma_{1} z_{1} \sigma_{2} z_{2} \ldots z_{n} \sigma_{2} y
$$

and, by repeating part of one of these sequences if necessary, we may assume that $m=n$. Since $\left(\sigma_{1}, P_{1}, \tau_{1}\right)$ and $\left(\sigma_{2}, P_{2}, \tau_{2}\right)$ are congruences on $V$, it follows that ax $\sigma_{1} c_{1} z_{1} \sigma_{2} c_{2} z_{2} \ldots c_{n} z_{n} \sigma_{2} b y$. Therefore $a x \sigma b y$ and analogously $x a \sigma y b$.

This completes the proof that $(\sigma, P, \tau)$ is an admissible triple.

4. $v_{i} \subseteq v=\mathscr{C}(\sigma, P, \tau)$ for $i=1,2$. In view of symmetry, we show only that $v_{1} \subseteq v$. To this end, we apply Lemma 3.1. Parts (i) and (ii) of this lemma are trivially satisfied. Since $P$ is saturated by $\tau_{1} \vee \tau_{2}$, it is also saturated by $\tau_{1}$ which shows that part (iii) of the lemma holds; part (iv) holds trivially. By Lemma 3.1, we conclude that $v_{1} \subseteq v$.

5. Let $v_{3} \in \mathscr{C}(V)$ be such that $v_{1} \subseteq v_{3}$ and $v_{2} \subseteq v_{3}$. Then $v \subseteq v_{3}$. Indeed, let $v_{3}=\mathscr{C}\left(\sigma_{3}, P_{3}, \tau_{3}\right)$. We shall again apply Lemma 3.1. The hypothesis implies that

(i) $\sigma_{1}, \sigma_{2} \subseteq \sigma_{3}$,

(ii) $P_{1}, P_{2} \subseteq P_{3}$,

(iii) both $\tau_{1}$ and $\tau_{2}$ saturate $P_{3}$,

(iv) $\tau_{1 \mid \tau P_{3}}, \tau_{2 \mid \pi P_{3}} \subseteq \tau_{\left.3\right|_{n P_{3}}}$.

It follows that $\sigma=\sigma_{1} \vee \sigma_{2} \subseteq \sigma_{3}$ and $P_{1} \cup P_{2} \subseteq P_{3}$. Since both $\tau_{1}$ and $\tau_{2}$ saturate $P_{3}$, so does $\tau_{1} \vee \tau_{2}$. But then $P_{1} \cup P_{2} \subseteq P_{3}$ implies that

$$
P=\left(P_{1} \cup P_{2}\right)\left(\tau_{1} \vee \tau_{2}\right) \subseteq P_{3} .
$$

In particular, $\tau$ saturates $P_{3}$. Let

$$
a_{1} \tau_{1} a_{2} \tau_{2} a_{3} \ldots a_{n-1} \tau_{2} a_{n}, \quad a_{1}, a_{n} \in T \backslash P_{3} .
$$

Now $a_{1} \in T \backslash P_{3}$ and the hypothesis (iv) above implies that $a_{2} \in T \backslash P_{3}$. But then the same hypothesis yields that $a_{3} \in T \backslash P_{3}$. Continuing this process till $a_{n}$, we see that in (3) all $a_{i} \in T \backslash P_{3}$. Therefore $\left(\tau_{1} \vee \tau_{2}\right)_{\mid \cap P_{3}} \subseteq \tau_{3 \mid \lambda P_{3}}$ and thus $\tau_{\mid \pi P_{3}} \subseteq \tau_{3 \mid \lambda P_{3}}$. We have verified the four conditions in Lemma 3.1 for the pair $v, v_{3}$ which then proves that $v \subseteq v_{3}$.

Therefore $v=v_{1} \vee v_{2}$, as asserted.

4. Restriction to the ideal $S$. The main result here is that the mapping $v \rightarrow v_{\mid S}$ is a complete homomorphism of $\mathscr{C}(V)$ onto $\mathscr{C}(S)$. Hence the induced congruence on $\mathscr{C}(V)$ has all its classes intervals; we provide for them explicit expressions. We also consider the mapping which to each congruence $v$ on $V$ associates the lower end of the corresponding interval. The section ends with a brief discussion of extendability of congruences on $S$ to all of $V$.

NOTATION 4.1. Let

$$
\mathscr{C}(S: V)=\{\sigma \in \mathscr{C}(S) \mid a \in V, x \sigma y \Rightarrow a x \sigma a y, x a \sigma y a\} .
$$

Lemma 4.2. If $\sigma \in \mathscr{C}(S: V)$, then $\left(\sigma,\{0\}, \varepsilon_{T}\right)$ is an admissible triple. Conversely if $(\sigma, P, \tau)$ is an admissible triple, then $\sigma \in \mathscr{C}(S: V)$.

Proof. The straightforward argument is omitted.

Corollary 4.3. Let $\sigma \in \mathscr{C}(S)$. Then $\sigma \in \mathscr{C}(S: V)$ if and only if there exists $v \in \mathscr{C}(V)$ such that $v_{\left.\right|_{S}}=\sigma$. 
LEMMA 4.4. Let $\sigma \in \mathscr{C}(S: V)$. Then $P_{\sigma}=\left\{a \in T^{*} \mid a\right.$ is $\sigma$-linked to some element in $\left.S\right\}$ $\cup\{0\}$ is an ideal of $T$. On $T / P$ define a relation $\sigma^{\prime}$ by

$$
a \sigma^{\prime} b \Leftrightarrow a x \sigma b y, x a \sigma y b \text { for all } x \sigma y\left(a, b \in T \backslash P_{\sigma}\right)
$$

and we define $0 \sigma^{\prime} 0$. Then $\sigma^{\prime}$ is an equivalence relation on $T / P_{\sigma}$. Let $\bar{\sigma}=\left(\sigma^{\prime}\right)^{\circ}$. Then $\bar{\sigma} \in \mathscr{C}_{0}\left(T / P_{\sigma}\right)$.

Proof. Let $a \in\left(P_{\sigma}\right)^{*}$ and $b \in T^{*}$ be such that $a b \notin 0$ in $T$. Then $a$ is $\sigma$-linked to some element $a^{\prime}$ in $S$. Using the hypothesis that $\sigma \in \mathscr{C}(S: V)$, we obtain, for any $x \in S$,

$$
(a b) x=a(b x) \sigma a^{\prime}(b x)=\left(a^{\prime} b\right) x, \quad x(a b)=(x a) b \sigma\left(x a^{\prime}\right) b=x\left(a^{\prime} b\right)
$$

so that $a b$ and $a^{\prime} b$ are $\sigma$-linked. Therefore $a b \in P_{\sigma}$ and dually $b a \in P_{\sigma}$. Consequently $P_{\sigma}$ is an ideal of $T$ and $T / P_{\sigma}$ is defined.

The hypothesis that $\sigma \in \mathscr{C}(S: V)$ implies that $\sigma^{\prime}$ is reflexive. It is obviously symmetric. If $a \sigma^{\prime} b$ and $b \sigma^{\prime} c$ for $a, b, c \in T \backslash P_{\sigma}$, then for any $x \sigma y$, we have ax $\sigma b y \sigma c y$ and $x a \sigma y b \sigma y c$ so that $a \sigma^{\prime} c$ and $\sigma^{\prime}$ is also transitive. Therefore $\sigma^{\prime}$ is an equivalence relation on $T / P$ and $\bar{\sigma}=\left(\sigma^{\prime}\right)^{\circ}$ is defined. Since $\sigma^{\prime}$ is 0 -restricted and $\bar{\sigma} \subseteq \sigma^{\prime}$, it follows that also $\bar{\sigma}$ is 0 -restricted. Therefore $\bar{\sigma} \in \mathscr{C}_{0}\left(T / P_{\sigma}\right)$.

We are now ready for the principal result of this section.

THEOREM 4.5. The mapping

$$
\chi: v \rightarrow v_{\left.\right|_{s}} \quad(v \in \mathscr{C}(V))
$$

is a complete homomorphism of $\mathscr{C}(V)$ onto $\mathscr{C}(S: V)$ which induces the complete congruence $R$ defined by

$$
\lambda R \rho \Leftrightarrow \lambda_{\mid s}=\rho_{\mid s} \quad(\lambda, \rho \in \mathscr{C}(V)) .
$$

For $v=\mathscr{C}(\sigma, P, \tau)$, we have $v R=\left[v_{R}, v^{R}\right]$ where $v_{R}=\mathscr{C}\left(\sigma,\{0\}, \varepsilon_{T}\right), v^{R}=\mathscr{C}\left(\sigma, P_{\sigma}, \bar{\sigma}\right)$.

Proof. Let $\mathscr{F} \subseteq \mathscr{C}(V)$. We must show that

$$
\left.\left(\bigwedge_{v \in \mathscr{F}} v\right)\right|_{S}=\bigwedge_{v \in \mathscr{F}}\left(\left.v\right|_{S}\right),\left.\quad\left(\bigvee_{v \in \mathscr{F}} v\right)\right|_{S}=\bigvee_{v \in \mathscr{F}}\left(\left.v\right|_{S}\right)
$$

For any $\rho \in \mathscr{F}$, we have $\bigwedge_{v \in \mathscr{F}} v \subseteq \rho$ so that $\left.\left.\left(\bigwedge_{v \in \mathscr{F}} v\right)\right|_{s} \subseteq \rho\right|_{s}$ and thus $\left.\left(\bigwedge_{v \in \mathscr{F}} v\right)\right|_{s} \subseteq \bigwedge_{v \in \mathscr{F}}\left(\left.v\right|_{s}\right)$. Conversely, let $(a, b) \in \bigwedge_{v \in \mathscr{F}}\left(\left.v\right|_{s}\right)$. Then for all $v \in \mathscr{F},\left.(a, b) \in v\right|_{s}$ so that $a v b$ and thus $(a, b) \in \bigwedge_{v \in \mathscr{F}} v$ and finally $\left.(a, b) \in\left(\bigwedge_{v \in \mathscr{F}} v\right)\right|_{s}$. This proves the first formula in (4).

Let $\left.(a, b) \in\left(\bigvee_{v \in \mathscr{F}} v\right)\right|_{s}$. Then $(a, b) \in \bigvee_{v \in \mathscr{F}} v$ which implies the existence of a sequence of

the form

$$
\text { a } v_{1} c_{1} v_{2} c_{2} v_{3} \ldots c_{n-1} v_{n} b
$$

for some $c_{1}, c_{2}, \ldots, c_{n-1} \in V$ and $v_{1}, v_{2}, \ldots, v_{n} \in \mathscr{F}$. For any $x \in S$, we obtain

$$
\text { ax } v_{1} c_{1} x v_{2} c_{2} x v_{3} \ldots c_{n-1} x v_{n} b x
$$

where $c_{1} x, c_{2} x, \ldots, c_{n-1} x \in S$. Letting $\sigma_{i}=v_{\left.i\right|_{s}}$ for $i=1,2, \ldots, n$ and $\sigma=\sigma_{1} \vee \sigma_{2} \vee$ 
$\ldots \vee \sigma_{n}$ we obtain $a x \sigma b x$. Dually one shows that $x a \sigma x b$. Since $x \in S$ is arbitrary and $S / \sigma$ is weakly reductive, it follows that $a \sigma b$. But then $(a, b) \in \bigvee_{v \in \mathscr{Y}}\left(\left.v\right|_{s}\right)$ which proves that $\left.\left(\bigvee_{v \in \mathscr{F}} v\right)\right|_{s} \subseteq \bigvee_{v \in \mathscr{F}}\left(\left.v\right|_{s}\right)$. Conversely, for every $\rho \in \mathscr{F}, \bigvee_{v \in \mathscr{F}} v \supseteq \rho$ so that $\left.\left(\bigvee_{v \in \mathscr{F}} v\right)\right|_{\left.s \supseteq \rho\right|_{s} \text { and }}$ thus $\left.\left(\bigvee_{v \in \mathscr{F}} v\right)\right|_{s} \supseteq \bigvee_{v \in \mathscr{F}}\left(\left.v\right|_{s}\right)$. This proves the second formula in (4).

That $\chi$ maps $\mathscr{C}(V)$ onto $\mathscr{C}(S: V)$ is a direct consequence of Corollary 4.3. Trivially $\chi$ induces $R$ on $\mathscr{C}(V)$ which is then a complete congruence.

Let $v=\mathscr{C}(\sigma, P, \tau)$. Then $v_{\mid s}=\sigma$ so that $\sigma \in \mathscr{C}(S: V)$. This implies that $\left(\sigma,\{0\}, \varepsilon_{T}\right)$ is an admissible triple which obviously gives that $\mathscr{C}\left(\sigma,\{0\}, \varepsilon_{T}\right)$ is the least element of $v R$. Lemma 4.4 clearly implies that $\left(\sigma, P_{\sigma}, \bar{\sigma}\right)$ is an admissible triple. Since its definition depends only upon $\sigma$, for the maximality of $v^{R}$, it suffices to show that $v \subseteq v^{R}$. To this end, we apply Lemma 3.1. Condition (i) of that lemma is trivially fulfilled. Condition (ii) holds by the definition of $P_{\sigma}$. For condition (iii), we let $a \in P_{\sigma}$ and $b \in T^{*}$ be such that $a \tau b$. By hypothesis there exists $a^{\prime} \in S$ which is $\sigma$-linked to $a$. For any $x \in S$, we have $a^{\prime} x \sigma a x \sigma b x$ and $x a^{\prime} \sigma x a \sigma x b$ and thus $b$ and $a^{\prime}$ are $\sigma$-linked. Hence $b \in P_{\sigma}$ and condition (iii) holds. For condition (iv), let $a, b \in T \backslash P_{\sigma}$ be such that $a \tau b$. For any $u, v \in(T / P)^{1}$, we have $u a v \tau u b v$ so that $u a v \neq 0$ if and only if $u b v \neq 0$ in $T / P$. Assume that $u a v \neq 0$. Then for any $x \sigma y$, we have (uav) $x \sigma(u b v) y$ and $x(u a v) \sigma y(u b v)$. It follows that $u a v \sigma^{\prime} u b v$ in Lemma 4.4. But then $a\left(\sigma^{\prime}\right)^{\circ} b$, that is $a \bar{\sigma} b$. This verifies condition (iv). By Lemma 3.1, we conclude that $v \subseteq v^{R}$.

Since trivially $v^{R} \in v R$ and $v R$ is convex, it follows that $v R=\left[v_{R}, v^{R}\right]$, as asserted.

Note that the congruence property (without completeness) of $R$ also follows from

COROLlary 4.6. $\mathscr{C}(S: V)$ is a complete sublattice of $\mathscr{C}(S)$ with least element $\varepsilon_{S}$ and greatest element $\omega_{s}$.

We now briefly explore the behaviour of the mapping $v \rightarrow v_{R}$.

LemMa 4.7. The mapping

$$
\psi: \sigma \rightarrow \mathscr{C}\left(\sigma,\{0\}, \varepsilon_{T}\right) \quad(\sigma \in \mathscr{C}(S: V))
$$

is an isomorphism of $\mathscr{C}(S: V)$ onto $\left\{v_{R} \mid v \in \mathscr{C}(V)\right\}$ which is a complete sublattice of $\mathscr{C}(V)$.

Proof. Let $\mathscr{F} \subseteq \mathscr{C}(S: V)$. In view of Theorem 4.5, it suffices to prove

and

$$
\bigwedge_{\sigma \in \mathscr{F}} \mathscr{C}\left(\sigma,\{0\}, \varepsilon_{T}\right)=\mathscr{C}\left(\bigwedge_{\sigma \in \mathscr{F}} \sigma,\{0\}, \varepsilon_{T}\right)
$$

$$
\bigvee_{\sigma \in \mathscr{F}} \mathscr{C}\left(\sigma,\{0\}, \varepsilon_{T}\right)=\mathscr{C}\left(\bigvee_{\sigma \in \mathscr{F}} \sigma,\{0\}, \varepsilon_{T}\right)
$$

By Corollary 4.6, $\mathscr{C}(S: V)$ is a complete sublattice of $\mathscr{C}(V)$ which implies that both $\bigwedge_{\sigma \in \mathscr{F}} \sigma$ and $\bigvee_{\sigma \in \mathscr{F}} \sigma$ are in $\mathscr{C}(S: V)$ and thus $\left(\bigwedge_{\sigma \in \mathcal{F}} \sigma,\{0\}, \varepsilon_{T}\right)$ and $\left(\bigvee_{\alpha \in \mathscr{F}} \sigma,\{0\}, \varepsilon_{T}\right)$ are admissible triples. It is clear that $\mathscr{C}\left(\bigwedge_{\sigma \in \mathscr{F}} \sigma,\{0\}, \varepsilon_{T}\right)$ is the greatest congruence contained in $\mathscr{C}\left(\sigma^{\prime},\{0\}, \varepsilon_{T}\right)$ for all $\sigma^{\prime} \in \mathscr{F}$ and $\mathscr{C}\left(\bigvee_{\sigma \in \mathscr{F}} \sigma,\{0\}, \varepsilon_{T}\right)$ is the least congruence containing 
$\mathscr{C}\left(\sigma^{\prime},\{0\}, \varepsilon_{T}\right)$ for all $\sigma^{\prime} \in \mathscr{F}$. This proves relations (5) and thus also the assertion of the lemma.

COROLlary 4.8. The mapping $v \rightarrow v_{R}(v \in \mathscr{C}(V))$ is a complete homomorphism of $\mathscr{C}(V)$ onto $\left\{v_{R} \mid v \in \mathscr{C}(V)\right\}$.

Proof. This follows directly from Theorem 4.5 and Lemma 4.7.

Heretofore the overall hypothesis has been that all congruences on $S$ be weakly reductive. For extendibility of congruences on $S$ to all of $V$ we need a stronger concept.

A semigroup $Q$ is reductive if for any $a, b \in Q, a x=b x$ for all $x \in Q$ implies $a=b$ and $x a=x b$ for all $x \in Q$ implies $a=b$. We say that a congruence $\rho$ on $Q$ is reductive if $Q / \rho$ is reductive.

Leмma 4.9. Every reductive congruence on $S$ can be extended to $V$.

Proof. Let $\sigma \in \mathscr{C}(S)$ be reductive, $a \in V$ and $x \sigma y$. Then for any $z \in S$, we have $z(a x)=(z a) x \sigma(z a) y=z(a y)$. By reductivity of $\sigma$, we conclude that $a x \sigma a y$ and dually $x a \sigma y a$. Hence $\sigma \in \mathscr{C}(S: V)$, so the assertion follows by Corollary 4.3.

We now consider some sufficient conditions on a semigroup in order that all its congruences be reductive. To this end, we first prove the following simple result.

LEMMA 4.10. Every inverse semigroup $S$ is reductive.

Proof. Let $a, b \in S$ be such that $a x=b x$ for all $x \in S$. Then $a\left(a^{-1} a\right)=b\left(a^{-1} a\right)$ and thus $a \leq b$. Similarly $b \leq a$ and so $a=b$ as required. Similarly $x a=x b$ for all $x \in S$ implies $a=b$.

Proposition 4.11. If $S$ is a monoid or an inverse semigroup, then every congruence on $S$ can be extended to a congruence on $V$.

Proof. The property of being a monoid obviously implies reductivity and carries over to homomorphic images. By Lemma 4.10, every inverse semigroup $S$ is reductive and by [3, Lemma II.1.10] all homomorphic images of $S$ are also inverse semigroups. The assertion now follows by Lemma 4.9.

5. The case when $V$ is regular and $S$ is completely 0 -simple. In such a case, we shall see that for any ideal $P$ of $T$, there exists a least congruence on $V$ of the form $(-, P,-)$.

Henceforth we assume that $V$ is a regular semigroup. We shall emphasize this by stating it explicitly in some statements but the hypothesis holds throughout. Note that this is equivalent to both $S$ and $T$ being regular. On any regular semigroup $Q$ we have the natural partial order:

$$
a \leq b \Leftrightarrow a=e b=b f \text { for some } e, f \in E(Q) .
$$

Lemma 5.1. Let $V$ be regular, $S$ be completely 0 -simple and $a \in T^{*}$ be such that $a S \neq 0$. Then there exists $b \in S^{*}$ such that $a>b$. Moreover, if $a \in E\left(T^{*}\right)$, we can find such $b \in E\left(S^{*}\right)$.

Proof. Let $v \in S$ be such that $a v \neq 0$. Since $S$ is regular, we have $a v=a v u a v$ for some $u \in S$ and thus $u a, a v \neq 0$. Let $s \in S$ be such that avsua $\neq 0$ and let $p$ be an inverse of 
avsua. Then for $e=a v s u a p, f$-vsuapa and $b=a v s u a p a$, we obtain

$$
\begin{gathered}
e^{2}=\operatorname{avsua}(\text { pavsuap })=\text { avsuap }=e, \quad f^{2}=v s u a(\text { pavsuap }) a=v s u a p a=f, \\
b=e a=a f \neq 0
\end{gathered}
$$

so that $a>b$ with $b \in S^{*}$. If $a \in E\left(T^{*}\right)$, then $b^{2}=$ avsua(paavsuap) $a=$ avsuapa $=$ $b \in E\left(S^{*}\right)$.

Lemma 5.2. Let $V$ be regular, $S$ completely 0 -simple, $v=\mathscr{C}(\sigma, P, \tau), a \in P^{*}, b \in S^{*}$, and $a>b$. Then $a v b$.

Proof. By hypothesis $b=e a=a f$ for some $e, f \in E(V)$. Let $b^{-1}$ be an inverse of $b$. Then

$$
\left(b b^{-1} e\right)^{2}=b b^{-1} e(e a) b^{-1} e=b b^{-1}(e a) b^{-1} e=b b^{-1} e \in E(S)
$$

and $b=\left(b b^{-1} e\right) a$. Hence we may assume that $e$ and similarly $f$ are elements of $S$. Also, by hypothesis, $a$ is $\sigma$-linked to some element $a^{\prime}$ of $S$. Hence $b=e a \sigma e a^{\prime}$ and $b=a f \sigma a^{\prime} f$ which implies that $b \sigma \leq a^{\prime} \sigma$. If $b \sigma=0$, then $\sigma=\omega_{s}$ and hence $a^{\prime} \sigma=0$. Otherwise $0 \neq b \sigma \leq a^{\prime} \sigma$ in the completely 0-simple semigroup $S / \sigma$ implies that $b \sigma=a^{\prime} \sigma$. Consequently $b \sigma a^{\prime}$ whence $b v a$.

Lemma 5.3. Let $V, S$ and $v$ be as in Lemma 5.2 and $a \in P^{*}$ be such that $a S=0$. Then $a v 0$.

Proof. If $s a \neq 0$ for some $s \in S$, then $s a=(s a) u(s a)$ for some $u \in S$ whence $a u \neq 0$, contrary to the hypothesis. Therefore $S a=0$. By hypothesis $a$ has a $\sigma$-linked element $a^{\prime}$ in $S$. Hence for any $x \in S$, we have $a x \sigma a^{\prime} x$ and $x a \sigma x a^{\prime}$ whence $a^{\prime} x \sigma x a^{\prime} \sigma 0$. Since $\sigma$ is weakly reductive, it follows that $a^{\prime} \sigma 0$. But then $a v a^{\prime} v 0$ so that $a v 0$.

The above two lemmas show which identifications take place in the case when $S$ is completely 0 -simple for every congruence which does not saturate $S$. They make it possible to prove the following result.

TheORem 5.4. Let $V$ be regular, $S$ completely 0 -simple and $P$ an ideal of $T$. The congruence $K_{P}$ generated by the relation

$$
\left\{(a, b) \in P^{*} \times S^{*} \mid a>b\right\} \cup\left\{(a, 0) \mid a \in P^{*}, a S=0\right\}
$$

is the least congruence on $V$ of the form $\mathscr{C}(\sigma, P, \tau)$ for some $\sigma, \tau$.

Proof. There exists at least one congruence $v$ of the form $\mathscr{C}(\sigma, P, \tau)$ namely $\mathscr{C}\left(\omega_{S}, P, \varepsilon_{T / P}\right)$. Let $v=\mathscr{C}(\sigma, P, \tau)$ be arbitrary. By Lemmas 5.2 and 5.3 , the relation in (6) is contained in $v$ and thus $\kappa_{P} \subseteq v$. Now $\kappa_{P}=\mathscr{C}\left(\sigma^{\prime}, P^{\prime}, \tau^{\prime}\right)$ for some $\sigma^{\prime}, P^{\prime}, \tau^{\prime}$. Lemma 3.1 implies that $P^{\prime} \subseteq P$.

Let $a \in P^{*}$. If $a>b$ for some $b \in S^{*}$, then $a K_{p} b$ by Lemma 5.2 and hence $a \in P^{\prime}$. If $a S=0$, then $a K_{P} 0$ by Lemma 5.3 and thus $a \in P^{\prime}$. By Lemma 5.1, there are no other possibilities.

Recall that $\pi_{A}$ denotes the principal congruence relative to a set $A$.

Corollary 5.5. Let $V, S, P$ and $\kappa_{P}$ be as in Theorem 5.4. Then

$$
\{\mathscr{C}(\sigma, P, \tau) \in \mathscr{C}(V) \mid \text { some } \sigma, \tau\}=\left[\kappa_{P}, \pi_{S \cup P^{*}}\right]
$$

and $\pi_{S \cup P^{*}}=\mathscr{C}\left(\omega_{S}, P, \xi_{T / P}\right)$. 
Proof. Any $\mathscr{C}(\sigma, P, \tau)$ saturates $S \cup P^{*}$ and $\pi_{S \cup P^{*}}$ is the greatest such. The last assertion follows easily from the definition of a principal congruence.

We shall now see that for an $S$ which is not completely 0 -simple, there may not exist the least congruence of the form $\mathscr{C}(\sigma, P, \tau)$ for a fixed $P$.

EXAMPLE 5.6. Let $V$ be the semilattice consisting of the infinite chain $S=\left\{\beta_{1}<\beta_{2}<\right.$ $\ldots\}$ and an element $\alpha$ greater than all the elements of $S$. Let $k \geq 1$ and $\pi$ be a partition of the interval $\left[\beta_{1}, \beta_{k}\right]$. Let $\rho_{\pi}$ be the partition of $V$ with classes; $\pi$-classes and $\left[\beta_{k+1}, \alpha\right]$. Then $\rho_{\pi}$ is a congruence on $V$ which does not saturate $S$. It is easy to see that all congruences on $V$ which do not saturate $S$ can be so constructed. Clearly $\wedge \rho_{\pi}=\varepsilon_{V}$.

Now consider $V$ as an extension of $S$ by $T=\{\alpha, 0\}$. The above shows that the set of all congruences on $V$ of the form $\mathscr{C}\left(\sigma, T, \varepsilon_{T / T}\right)$ for some $\sigma \in \mathscr{C}(S)$ does not have the least element.

The greatest element of the set of all congruences $\mathscr{C}(\sigma, P, \tau)$ for a fixed $P$ always exists and is equal to $\pi_{S \cup P^{*}}=\mathscr{C}\left(\omega_{S}, P, \xi_{T / P}\right)$.

\section{REFERENCES}

1. A. H. Clifford, Extensions of semigroups, Trans. Amer. Math. Soc. 68 (1950), 165-173.

2. M. Petrich, Congruences on extensions of semigroups, Duke Math. J. 34 (1967), 215-224.

3. M. Petrich, Inverse semigroups (Wiley-Interscience, New York, 1984).

4. M. Petrich, The congruence lattice of an extension of completely 0 -simple semigroups (preprint).

Dipartimento di Matematice

UNIVERSITÀ DI LECCE

73160 LECCE

ITALY
Present address:

Department of Mathematics

Simon Fraser University

Burnaby

British Columbia V5A 1S6 